\title{
Testing a Dilaton Gravity Model Using Nucleosynthesis
}

\author{
S. Boran and E. O. Kahya \\ Department of Physics, İstanbul Technical University, Maslak, 34469 Istanbul, Turkey \\ Correspondence should be addressed to S. Boran; borans@itu.edu.tr \\ Received 7 July 2014; Revised 6 August 2014; Accepted 7 August 2014; Published 27 August 2014 \\ Academic Editor: Elias C. Vagenas
}

Copyright (C) 2014 S. Boran and E. O. Kahya. This is an open access article distributed under the Creative Commons Attribution License, which permits unrestricted use, distribution, and reproduction in any medium, provided the original work is properly cited. The publication of this article was funded by SCOAP ${ }^{3}$.

Big bang nucleosynthesis (BBN) offers one of the most strict evidences for the $\Lambda$-CDM cosmology at present, as well as the cosmic microwave background (CMB) radiation. In this work, our main aim is to present the outcomes of our calculations related to primordial abundances of light elements, in the context of higher dimensional steady-state universe model in the dilaton gravity. Our results show that abundances of light elements (primordial $\mathrm{D},{ }^{3} \mathrm{He},{ }^{4} \mathrm{He}, \mathrm{T}$, and ${ }^{7} \mathrm{Li}$ ) are significantly different for some cases, and a comparison is given between a particular dilaton gravity model and $\Lambda$-CDM in the light of the astrophysical observations.

\section{Introduction}

The current expansion of the universe is a crucial evidence for the big bang cosmology model. It predicts the chemical abundances of primordial elements as a result of nuclear reactions which began seconds after the big bang and continued for the next several minutes. With the help of inflation, one can consistently solve the well-known problems of the standard model, such as the observed spatial homogeneity, isotropy, and flatness of the universe [1].

There are still many unsolved puzzles of this model, such as the origin of dark matter and dark energy, cosmological constant problem, cosmic coincidence problem, and the exact form of the inflation potential $[2,3]$. On the other hand, there are many models which claim solutions to these problems by modifying Einstein's general relativity. Quintessence, $k$ essence, phantom, quintom, and other phenomenological models are just few examples of alternate gravity models that offer a solution to the dark energy problem [4]. And also there are alternative gravity theories that suggest using extra fields (scalar tensor, etc.) and higher dimensions (KaluzeKlein, Randall-Sundrum) arising from string theory at the low-energy limit [5].

In this ocean of models, we would like to consider an observable consequence (modified abundances of light elements) of a new model, a higher dimensional dilaton gravity theory of steady-state cosmological (HDGS) model in the context of string theory. We need to highlight that the original steady-state model $[6,7]$ is unfavorable compared to the standard big bang scenario. But our motivation in this work is to suggest a test of a specific higher dimensional dilaton gravity model which effectively mimics the standard FRW model with the modified Hubble constant. Hence, an immediate consequence would be the modification of nucleosynthesis.

This modification was investigated in [8] and it was claimed that this model gives a better estimate for the primordial ${ }^{4} \mathrm{He}$ abundance compared to the standard big bang nucleosynthesis (SBBN) by choosing the number of dimensions appropriately. In this work, to further test their strong claim, we calculated the abundances of the primordial $\mathrm{D},{ }^{3} \mathrm{He},{ }^{4} \mathrm{He}, \mathrm{T}$, and ${ }^{7} \mathrm{Li}$ in the context of this nonstandard (HDGS) model and compared it with the predictions of SBBN and the astrophysical observations.

On the other hand, at high energies, the quantum gravitational corrections will start to play an important role. Quantum corrections will modify the dilaton gravity models as well and therefore change the whole form of this model via action [9-12]. One would naturally expect to see quantum effects during the very early universe such as primordial inflationary stage. During inflation, quantum loop effects may lead to very small $[13,14]$ but possibly observable corrections 
to power spectrum [15-20]. Therefore, one might describe the interactions with effective field theories of inflation [21]. But, in this work, we are mainly interested in the consequence of a geometrical constraint: $3+n$-dimensional universe having a constant volume, leading to a modified Hubble parameter during a later stage, nucleosynthesis, where quantum gravitational corrections are negligible.

The paper is organized as follows. In Section 2, dynamics of this particular dilaton gravity model is summarized. In Section 3, nucleosynthesis in the context of this model is analyzed. In Section 4, the results obtained from our calculations for this model and the predictions of SBBN with the help of Planck satellite data [22] are compared with the astrophysical observations.

\section{Dynamics of HDGS: A Dilaton Gravity Model}

In this section, we briefly summarize the dynamics of a particular type of dilaton gravity models proposed in [8]. The idea is introducing a higher dimensional dilaton gravity action of steady-state cosmology (HDGS) in the string frame. Therefore, the evolution of the internal $n$-dimensional space results in an evolution of the observed universe to keep the whole system in a steady state. Due to this constraint, choosing particular values, for some parameters, such as the number of extra dimensions, leads to possibly observable effects in our universe. Let us start with the action, which stems from the low-energy effective string theory,

$$
S=\int_{M} d^{1+3+n} x \sqrt{|g|} e^{-2 \phi}\left(R+4 \omega \partial_{\mu} \phi \partial^{\mu} \phi+U(\phi)\right)
$$

where $R$ is the curvature scalar, $M$ stands for manifold, $n$ corresponds to extra dimensions, $|g|$ is the determinant of $g_{\mu \nu}$ metric tensor, $\phi$ is the dilaton field taken as space independent real function of time, and $\omega$ is an arbitrary coupling constant. $U(\phi)=U_{0} e^{\lambda \phi}$ is a real smooth function of the dilaton field and corresponds to the dilaton self-interaction potential and both $U_{0}$ and $\lambda$ are real parameters. Two interesting cases that are worth mentioning are $\omega=1$ and $n=6$, corresponding to anomaly-free superstring theory and $\omega=1$ with $n=22$ corresponds to bosonic string theory. The metric is given by

$$
\begin{aligned}
d s^{2}= & -d t^{2}+a^{2}(t)\left(d x^{2}+d y^{2}+d z^{2}\right) \\
& +s^{2}(t)\left(d \theta_{1}^{2}+\cdots+d \theta_{n}^{2}\right) .
\end{aligned}
$$

Here, $t$ is the cosmic time and $(x, y, z)$ are the cartesian coordinates of the 3-dimensional flat space, basically the observed universe. The coordinates, $\theta$, are $n$-dimensional, compact (torodial) internal space coordinates (this represents space that cannot be observed directly and locally today). While $a(t)$ denotes the scale factor of 3-dimensional external space, $s(t)$ is the scale factor of $n$-dimensional internal space.

This model has the following key properties.

(i) The $(3+n)$-dimensional universe has a constant volume; that is, $V=a^{3} s^{n}=V_{0}$, hence steady state. But the internal and external spaces are dynamical. (ii) The energy density is constant in the higher dimensional universe. (iii) There is no higher dimensional matter source other than the dilaton field in the action.

If the scalar field is redefined as $\beta=e^{\lambda \phi}$, the relation between the scalar field and the scale factor of the external space turns out to be

$$
\left(\frac{a^{\prime}}{a}\right)^{2}=\frac{n}{3(3+n)} \frac{2 \omega \varepsilon}{\beta^{2}},
$$

where $\varepsilon$ is a constant of integration. Here, prime denotes derivative with respect to the ordinary time. Imposing the constant volume condition gives

$$
\begin{aligned}
& a=a_{0} e^{ \pm(1 / 3) \sqrt{3 n /(3+n)} \sqrt{2 \omega \varepsilon} \int d t(1 / \beta),} \\
& s=s_{0} e^{\mp(1 / n) \sqrt{3 n /(3+n)} \sqrt{2 \omega \varepsilon} \int d t(1 / \beta),}
\end{aligned}
$$

where $a_{0}$ and $s_{0}$ correspond to the integration constants. Therefore, the modified Hubble parameter of the external space is obtained as follows:

$$
H_{a} \equiv \frac{a^{\prime}}{a}= \pm \frac{1}{3} \sqrt{\frac{3 n}{3+n}} \frac{\sqrt{2 \omega \varepsilon}}{\beta} .
$$

Here, the physically relevant case is the solution for expanding external space with $H_{a}>0$. The deceleration parameter for the external space is given by

$$
q_{a} \equiv-\frac{a^{\prime \prime} a}{a^{\prime 2}}=-1 \pm 3 \sqrt{\frac{3+n}{3 n}} \frac{\beta^{\prime}}{\sqrt{2 \omega \varepsilon}} .
$$

In the case of $\varepsilon \neq 0$ and $U_{0} \neq 0$, and with the choice of appropriate initial conditions, it turns out that [8] the early time modified deceleration parameter is given by

$$
q \longrightarrow 3 \sqrt{\frac{3+n}{3 n}} \frac{1}{\sqrt{\omega}}-1 \text {. }
$$

\section{Nucleosynthesis in HDGS}

We are interested in how abundances of light elements would change in the context of this model. Specifically, we would like to consider the ratio of the modified expansion rate to the standard expansion rate during the early radiation dominant epoch. This ratio is given by

$$
S \equiv \frac{H_{a}}{H_{\mathrm{SBBN}}}=\frac{1+q_{\mathrm{SBBN}}}{1+q_{a}} .
$$

This is true, since deceleration parameter stays almost constant during primordial nucleosynthesis. The value of the deceleration parameter for standard $\mathrm{BBN}$ is $q_{\mathrm{SBBN}}=1$. Since $q_{a}$ is given by (7), the so-called standard expansion factor, $S$, can be expressed in terms of $\omega$ and $n$ as

$$
S=\frac{2}{3}\left(\sqrt{\frac{3 \omega n}{3+n}}\right) .
$$


If $S \neq 1$ is taken, it denotes nonstandard expansion factor. This kind of modification might also arise due to additional light particles such as neutrinos which would make the ratio be $\widetilde{H} / H_{\mathrm{SBBN}}=\left[1+(7 / 43)\left(N_{v}-3\right)\right]^{1 / 2}$. In this context of the dilaton gravity model that we mentioned, it is also going to occur due to a modification of general relativity. We are interested in the case where $N_{v}=3$ and therefore the value of $(S-1)$ will come only from the modification of general relativity.

The primordial abundances of the light elements (primordial $\mathrm{D},{ }^{3} \mathrm{He},{ }^{4} \mathrm{He},{ }^{7} \mathrm{Li}$, and $\mathrm{T}$ ) depend on the baryon density and the expansion rate of the universe $[23,24]$. The baryon density parameter [23] is given by

$$
\eta_{10} \equiv 10{ }^{10} \eta_{B} \equiv 10 \frac{10}{n_{\gamma}}=273.9 \Omega_{B} h^{2}
$$

where $\eta_{B}$ gives the baryon to photon ratio, $\Omega_{B}$ is dimensionless current critical cosmological density parameter for baryons, and $h=h_{100} \equiv H_{0} / 100 \mathrm{kms}^{-1} \mathrm{Mpc}^{-1}$ with $H_{0}$ being the present value of the Hubble parameter. Any modification of the expansion rate would change the time when neutrons freeze out, which will in turn determine the final abundance of helium- 4 as well as all of the other light elements.

In the following subsections, we will analyze nucleosynthesis due to a modification of the expansion rate in the context of HDGS models. We will express the primordial nuclear abundances of light nuclei in terms of two parameters of HDGS models: number of extra dimensions $n$ and coupling constant $\omega$. Particularly, we will be interested in the case of $\omega=1$, where $n=6$ and $n=22$ correspond to anomaly-free superstring and bosonic string theory, respectively.

3.1. ${ }^{4} \mathrm{He}$ Abundance in HDGS Models. The two body reaction chains of light elements, which include deuterium (D), tritium (T), and helium-3 $\left({ }^{3} \mathrm{He}\right)$ to produce helium-4 $\left({ }^{4} \mathrm{He}\right)$, are more efficient than four body reactions of neutrons and protons. The first step is producing $\mathrm{D}$ from $n+p \rightarrow \mathrm{D}+\gamma$. After that, $\mathrm{D}$ is converted into ${ }^{3} \mathrm{He}$ and $\mathrm{T}$ as follows:

$$
\mathrm{D}+\mathrm{D} \longrightarrow{ }^{3} \mathrm{He}+n \quad \mathrm{D}+\mathrm{D} \longrightarrow \mathrm{T}+p
$$

and, finally, ${ }^{4} \mathrm{He}$ is produced from $\mathrm{D}$ combining with $\mathrm{T}$ and ${ }^{3} \mathrm{He}$

$$
\mathrm{T}+\mathrm{D} \longrightarrow{ }^{4} \mathrm{He}+n \quad{ }^{3} \mathrm{He}+\mathrm{D} \longrightarrow{ }^{4} \mathrm{He}+p .
$$

In order to get precise estimates for abundances of light elements, one should solve nonlinear differential equations of the nuclear reaction network. This problem can be studied numerically and the modern methods are based on Wagoner et al. [25] code and its updated version by Smith et al. [26]. The next step is getting a best fit to a numerical work to see how various abundances depend on $\eta_{10}$ and other parameters such as number of extra neutrinos. Another venue is applying semianalytical methods, where one of the earliest works was done by Esmailzadeh et al. [27] using the method of fixed points.

In this work, we would like to use, if there exists, the best fit expressions for certain elements. If there is none in the literature for a certain element, then we will use a semianalytical approach that is based on a simple assumption, which is the nuclear reaction network obeying in a quasiequilibrium state. In this state, basically one assumes that "the total flux coming into each corresponding reservoir must be equal to the outgoing flux" [28].

A simple way of estimating of ${ }^{4} \mathrm{He}$ abundance (in general, abundance by weight is related to the ratio of number density of a particular element to the number density of all nucleons (including the ones in complex nuclei), $X_{A} \equiv A n_{A} / n_{N}$, where $A$ is the mass number of a particular element; e.g., $A=4$ for helium) is the following: multiply the abundance of neutrons by two at the time when the deuterium bottleneck opens up. Here, we will refer to the best fit expression for ${ }^{4} \mathrm{He}$ abundance that includes the case of modified expansion rate $[29,30]$ :

$$
Y_{p}=0.2485 \pm 0.0006+0.0016\left(\left(\eta_{10}-6\right)+100(S-1)\right)
$$

where $p$ stands for the primordial abundance. We will take $\eta_{10} \simeq 6$ [31] from here on. The SBBN value, $S=1$, becomes $Y_{p}^{\text {SBBN }}=0.2485 \pm 0.0006$. Using (9), for the case of HDGS models that we are interested in, one can get the following expression for ${ }^{4} \mathrm{He}$ abundance in terms of $\omega$ and $n$ as [8]

$$
Y_{p}=0.2485 \pm 0.0006+0.16\left(-1+\frac{2}{3} \sqrt{\frac{3 \omega n}{3+n}}\right)
$$

In the case of $\omega=1$, the predicted $Y_{p}$ values are obtained as $Y_{p}=0.2393 \pm 0.0006$ and $Y_{p}=0.2618 \pm 0.0006$, for $n=6$ and $n=22$, respectively.

From the observational point of view, the ${ }^{4} \mathrm{He}$ primordial abundance, $Y_{p}$, is determined from the recombination of lines of the H II from blue compact galaxies (BCGs) [32]. The observational results of the ${ }^{4} \mathrm{He}$ abundances are given by $Y_{p}=$ $0.2565 \pm 0.0060[33]$ and $Y_{p}=0.2561 \pm 0.0108$ [34]

\subsection{Abundances of Other Light Elements in HDGS Models}

3.2.1. Deuterium Abundance. Deuterium is produced by $p+$ $n \rightarrow \mathrm{D}+\gamma$ and used in four types of reactions (11), (12). Therefore, one would expect to solve either numerically or analytically the equations for this nuclear reaction network and get the expression for deuterium abundance, $X_{\mathrm{D}} \equiv$ $2 n_{\mathrm{D}} / n_{\mathrm{N}}$, where $n_{\mathrm{D}}$ and $n_{\mathrm{N}}$ are the number densities of deuterium and all nucleons, respectively.

In literature, instead of abundances of elements, their abundances relative to hydrogen are given. To see why, let us look at how deuterium is determined. The absorbed primordial element has more space in the wings of the observed quasar absorption-line systems (QAS) [35-39] than the absorbed hydrogen at high redshifts ( $\mathrm{z}$ ) and/or at low metallicity $(Z)$. Also, the observation of the multicomponent velocities of these absorbed elements is very significant in order to determine the abundance of deuterium. Therefore, the $(\mathrm{D} / \mathrm{H})_{p}$ ratio is more meaningful and is often known as interstellar medium measurement for deuterium abundance. 
This ratio can be expressed in terms of the abundance by weight of the deuterium as

$$
y_{\mathrm{D} p} \equiv 10^{5}\left(\frac{n_{\mathrm{D}}}{n_{\mathrm{H}}}\right)_{p}=10^{5}\left(\frac{13}{24} X_{\mathrm{D} p}\right) .
$$

The factor, $13 / 24$, comes from the fact that mass number of deuterium is 2 and hydrogen number density is equal to $12 / 13$ of all the nucleons in the universe, that is, $75 \%$ by weight.

Let us start with the semianalytical expression for the abundance of deuterium to calculate (15). Using the quasiequilibrium condition, one can get [28]

$$
X_{\mathrm{D} p} \simeq \frac{2 R}{\exp \left(A \eta_{10}\right)-1} \simeq 4.87 \times 10^{-5},
$$

where $R \simeq 2 \cdot 10^{-5}[28], \eta_{10} \simeq 6$, and $A \simeq 0.1$. Here, the coefficients $R$ and $A$ are related to experimental values of nuclear reaction rates of deuterium at temperature of order $0.08 \mathrm{MeV}$. (We assume that the nuclear interaction rates are independent of extra dimensions. We also assume that there are no matter sources in higher dimensions and HDGS is a Kaluza-Klein-type model rather than a brane world cosmology one.) Putting this value in (15) gives $y_{\mathrm{D} p}^{\mathrm{SBBN}}=2.63$.

Let us now use a more precise expression for deuterium abundance [23] based on a numerical best fit:

$$
y_{\mathrm{D} p}=2.60(1 \pm 0.06)\left(\frac{6}{\eta_{10}-6(S-1)}\right)^{1.6} .
$$

From this expression, one can get the SBBN value of $y_{\mathrm{D} p}$ (for $S=1$ and $\left.\eta_{10} \simeq 6\right)$ as $y_{\mathrm{D} p}^{\mathrm{SBBN}}=2.60 \pm 0.16$. Comparing this number with the one from the semianalytical method, $y_{\mathrm{D} p}^{\mathrm{SBBN}}=2.63$, we can safely assume a quasiequilibrium condition, if necessary.

By using (9), one can express $y_{\mathrm{D} p}$ for HDGS models as

$$
\begin{aligned}
& y_{\mathrm{D} p} \\
& =2.60(1 \pm 0.06)\left(\frac{6}{\eta_{10}-6(-1+(2 / 3) \sqrt{3 \omega n /(3+n)})}\right)^{1.6} .
\end{aligned}
$$

Taking $\eta_{10} \simeq 6$, the predicted values of $y_{\mathrm{D} p}$ are obtained as $y_{\mathrm{D} p}=2.38 \pm 0.16$ and $y_{\mathrm{D} p}=2.99 \pm 0.16$ for $n=6$ and $n=22$, respectively, for $\omega=1$ model.

Finally, the observational results are $y_{\mathrm{D} p}=2.87 \pm 0.22$ [40] and $y_{\mathrm{D} p}=2.54 \pm 0.05[39]$.

3.2.2. Helium-3 Abundance. The relevant nuclear reactions that involve ${ }^{3} \mathrm{He}$ are

$$
\begin{array}{cc}
\mathrm{D}+\mathrm{D} \longrightarrow{ }^{3} \mathrm{He}+n & \mathrm{D}+p \longrightarrow{ }^{3} \mathrm{He}+\gamma \\
{ }^{3} \mathrm{He}+n \longrightarrow \mathrm{T}+p & { }^{3} \mathrm{He}+\mathrm{D} \longrightarrow{ }^{4} \mathrm{He}+p .
\end{array}
$$

The quantity used in the literature to describe ${ }^{3} \mathrm{He}$ is

$$
y_{3} \equiv 10^{5}\left(\frac{n_{3} \mathrm{He}}{n_{\mathrm{H}}}\right)=10^{5}\left(\frac{13}{36} X_{{ }^{3} \mathrm{He}}\right) .
$$

Making a quasiequilibrium approximation for ${ }^{3} \mathrm{He}$ abundance, we can express the ${ }^{3} \mathrm{He}$ abundance in terms of deuterium abundance after using the experimental values for the ratios of the related nuclear reaction rates [28]

$$
X_{{ }^{3} \mathrm{He}} \simeq \frac{0.2 \cdot X_{\mathrm{D}}+10^{-5}}{1+4 \times 10^{3} X_{\mathrm{D}}} .
$$

From this equation, we can see that ${ }^{3} \mathrm{He}$ abundance is not as sensitive as deuterium since a change in deuterium abundance would change both parts of the ratio. One can also see this from the weaker dependence of $y_{3}$ on $\eta_{10}$, compared to $y_{\mathrm{D} p}$, for SBBN best fit expression [41]. Consider

$$
y_{3}=3.1(1 \pm 0.03) \eta_{10}^{-0.6} .
$$

Therefore, ${ }^{3} \mathrm{He}$ abundance is not a good indicator of a modification of SBBN due to HDGS models.

3.2.3. Tritium Abundance. Using the quasiequilibrium condition for tritium, $X_{T}{ }^{f}[28]$ is obtained as

$$
X_{T}^{f} \simeq\left(0.015+3 \cdot 10^{2} X_{3^{H e}}{ }^{f}\right) X_{\mathrm{D}}{ }^{f} .
$$

It is clear from this expression that the value of tritium abundance will be as sensitive as deuterium abundance to any modification of the expansion rate. But the magnitude of tritium abundance is two orders of magnitude smaller than both deuterium and helium-3. Therefore, observationally, it is not very feasible, but it should be kept in mind that it can be used to test for consistency in the future experiments.

3.2.4. Lithium-7 Abundance. Finally, we would like to investigate the effects of modified expansion rate on lithium abundance. The ${ }^{7} \mathrm{Li}$ abundance is given by

$$
y_{\mathrm{Lip}} \equiv 10^{10}\left(\frac{n_{\mathrm{Li}}}{n_{\mathrm{H}}}\right)_{p} .
$$

One might think that its smallness would make it irrelevant for observational purposes. But it can actually be measured in the atmospheres of metal-poor stars in the stellar halo of Milky Way. All primordial elements point towards the same $\eta_{10}$ parameter except lithium. The ratio of the expected SBBN value of lithium-7 abundance to the observed one is between 2.4 and 4.3 [42]. Therefore, it should be interesting to check if these HDGS models offer any solution to the lithium problem.

The best fit expression to the numerical BBN data of the $y_{\mathrm{Lip}}$ is given in [23] as

$$
y_{\text {Lip }}=4.82(1 \pm 0.10)\left(\frac{\eta_{10}-3(S-1)}{6}\right)^{2} .
$$

Taking $S=1$ and $\eta_{10} \simeq 6$, the SBBN value of lithium-7 abundance is found as $y_{\mathrm{Lip}}^{\mathrm{SBBN}}=4.82 \pm 0.48$. In terms of $\omega$ and $n$, the modified form of (25) becomes

$$
y_{\text {Lip }}=4.82(1 \pm 0.10)\left(\eta_{10}-3\left(-1+\frac{2}{3} \sqrt{\frac{3 \omega n}{3+n}}\right)\right)^{2} .
$$


TABLE 1: The abundances of He-4, deuterium, and Li-7 for different models.

\begin{tabular}{lccc}
\hline Models and data & $Y_{p}$ & $y_{D p}$ & $y_{\text {Lip }}$ \\
\hline SBBN model & $0.2485 \pm 0.0006$ & $2.60 \pm 0.16$ & $4.82 \pm 0.48$ \\
$n=6$ dilaton gravity model & $0.2393 \pm 0.0006$ & $2.38 \pm 0.16$ & $5.10 \pm 0.51$ \\
$n=$ 22 dilaton gravity model & $0.2618 \pm 0.0006$ & $2.99 \pm 0.16$ & $4.43 \pm 0.44$ \\
Observational data & $0.2561 \pm 0.0108[34]$ & $2.88 \pm 0.22[40]$ & $1.1-1.5[43]$ \\
& $0.2565 \pm 0.0060[33]$ & $2.54 \pm 0.05[39]$ & $1.23_{-0.32}^{+0.68[44]}$ \\
\hline
\end{tabular}

By using (26), the predicted $y_{\mathrm{Lip}}$ values are found as $y_{\mathrm{Lip}}=$ $5.10 \pm 0.51$ for $n=6$ and $y_{\text {Lip }}=4.43 \pm 0.44$ for $n=22$, for the case of $\omega=1$.

\section{Discussion}

We have shown in this work that one gets a considerable modification to the primordial abundances of light elements in the case of a higher dimensional steady-state universe in dilaton gravity (there are other ways to modify BBN based on scalar-tensor theories; for details, see [45-47] and references therein.). Although there is a huge class of models that one can consider, with two free parameters $\omega$ (dilaton coupling constant) and $n$ (number of internal dimensions). Here we focused on two interesting cases: $\omega=1$ with $n=6$ (anomalyfree superstring theory), and $\omega=1$ with $n=22$ (bosonic string theory).

The main idea behind the calculation is modifying the expansion rate during the nucleosynthesis to get different abundances for light elements. One can think of the modification as being similar to adding more relativistic particles, such as extra neutrinos, into the standard big bang model. When Hubble parameter gets modified, all the nucleosynthesis will get modified as well. The question is the following: is this modification large enough to observe and if it is then is it compatible with the data?

To answer these questions, one should analyze how the nuclear reactions get modified with the modification of the expansion rate. It is well-known that the complete analysis of the nuclear reactions governing the primordial abundances of light elements can be done using numerical methods. We used the results of the previous works, where we can, which were obtained by getting best fit expressions to numerical data related to the abundances of these elements. And if there are no known best fit expressions in the literature, we proceeded our analysis based on semianalytical methods.

The primordial abundance of helium-4 was already studied in the context of these models. It was pointed out that $n=22$ case is more compatible with helium- 4 data compared to the standard big bang scenario. We made a more extensive analysis of other light elements and checked the compatibility of this model with astrophysical observables. The results are summarized in Table 1.

One can clearly see from Table 1 that $\omega=1$ and $n=6$ dilaton gravity model is incompatible with helium-4 data and is incompatible with deuterium as well. Helium-4 data favoured the case of $\omega=1$ and $n=22$ compared to SBBN, as was noted. In the case of deuterium, earlier measurements favour (with almost being inside the error bars) dilaton gravity model whereas the more recent measurements rule them out and point towards SBBN. Therefore, it is fair to say that one needs more observations and data analysis to see which model is favoured.

We also showed that helium-3 and tritium abundances are not very convenient to see a modification of the standard model in the context of the dilaton gravity model considered here. And for the case of lithium-7 one gets almost a ten percent decrease for the expected abundance, compared to $\mathrm{SBBN}$, but it is still far from explaining the observed abundance. So, these models do not offer a solution to the lithium problem; therefore, the existence of this problem still preserves its place in the literature and leaves an open window to new physics.

\section{Conflict of Interests}

The authors declare that there is no conflict of interests regarding the publication of this paper.

\section{Acknowledgments}

The authors would like to thank Özgür Akarsu, Ali Kaya, and Subir Sarkar for the helpful discussions. This work was supported by TÜBİTAK-1001 Grant no. 112 T817.

\section{References}

[1] A. Linde, "Inflationary cosmology," in Inflationary Cosmology, vol. 738 of Lecture Notes in Physics, pp. 1-54, 2007.

[2] P. J. E. Peebles and B. Ratra, "The cosmological constant and dark energy," Reviews of Modern Physics, vol. 75, no. 2, pp. 559606,2003 .

[3] S. Perlmutter, G. Aldering, G. Goldhaber et al., "Measurements of $\Omega$ and $\Lambda$ from 42 high-redshift supernovae," The Astrophysical Journal, vol. 517, no. 2, pp. 565-586, 1999.

[4] R. Durrer and R. Maartens, "Dark energy and modified gravity," in Dark Energy: Observational and Theoretical Approaches, P. Ruiz-Lapuente, Ed., pp. 48-91, Cambridge University Press, 2010.

[5] L. Randall and R. Sundrum, "Large mass hierarchy from a small extra dimension," Physical Review Letters, vol. 83, no. 17, pp. 3370-3373, 1999.

[6] H. Bondi and T. Gold, "The steady-state theory of the expanding Universe," Monthly Notices of the Royal Astronomical Society, vol. 108, no. 3, pp. 252-270, 1948. 
[7] F. Hoyle, "A new model for the expanding universe," Monthly Notices of the Royal Astronomical Society, vol. 108, pp. 372-382, 1948.

[8] Ö. Akarsu and T. Dereli, "Late time acceleration of the 3-space in a higher dimensional steady state universe in dilaton gravity," Journal of Cosmology and Astroparticle Physics, vol. 2013, no. 2, article 050, 2013.

[9] S. Nojiri, O. Obregon, and S. D. Odintsov, "(Non)-singular brane-world cosmology induced by quantum effects in fivedimensional dilatonic gravity," Physical Review D, vol. 62, Article ID 104003, 2000.

[10] S. Nojiri and S. D. Odintsov, "Quantum dilatonic gravity in $\mathrm{d}=2,4$ and 5 dimensions," International Journal of Modern Physics A, vol. 16, no. 6, pp. 1015-1108, 2001.

[11] S. Nojiri and S. D. Odintsov, "Introduction to modified gravity and gravitational alternative for dark energy," International Journal of Geometric Methods in Modern Physics, vol. 4, no. 1, pp. 115-145, 2007.

[12] E. Elizalde, S. Jhingan, S. Nojiri, S. D. Odintsov, M. Sami, and I. Thongkool, "Dark energy generated from a (super-) string effective action with higher-order curvature corrections and a dynamical dilaton," The European Physical Journal C, vol. 53, no. 3, pp. 447-457, 2008.

[13] S. Wienberg, "Quantum contributions to cosmological correlations," Physical Review D, vol. 72, no. 4, Article ID 043514, 19 pages, 2005.

[14] S. Weinberg, "Quantum contributions to cosmological correlations. II. Can these corrections become large?" Physical Review D, vol. 74, Article ID 023508, 2006.

[15] D. Seery, "One-loop corrections to a scalar field during inflation," Journal of Cosmology and Astroparticle Physics, vol. 711, p. 25, 2007.

[16] D. Seery, "One-loop corrections to the curvature perturbation from inflation," Journal of Cosmology and Astroparticle Physics, vol. 2008, p. 006, 2008.

[17] D. Marolf and I. A. Morrison, "Infrared stability of de Sitter space: loop corrections to scalar propagators," Physical Review $D$, vol. 82, Article ID 105032, 2010.

[18] S. B. Giddings and M. S. Sloth, "Semiclassical relations and IR effects in de Sitter and slow-roll space-times," Journal of Cosmology and Astroparticle Physics, vol. 1101, article 023, 2011.

[19] E. O. Kahya and V. K. Onemli, "Quantum stability of a $w<-1$ phase of cosmic acceleration," Physical Review D, vol. 76, Article ID 043512, 2007.

[20] E. O. Kahya and R. P. Woodard, "Scalar field equations from quantum gravity during inflation," Physical Review D: Particles, Fields, Gravitation, and Cosmology, vol. 77, no. 8, 2008.

[21] C. Cheung, P. Creminelli, A. L. Fitzpatrick, J. Kaplan, and L. Senatore, "The effective field theory of inflation," Journal of High Energy Physics, vol. 2008, no. 3, article 014, 2008.

[22] P. A. R. Ade, N. Aghanim, C. Armitage-Caplan et al., "Planck 2013 results-XVI. Cosmological parameters," http://arxiv.org/abs/1303.5076.

[23] G. Steigman, "Neutrinos and big bang nucleosynthesis," Advances in High Energy Physics, vol. 2012, Article ID 268321, 24 pages, 2012.

[24] V. Simha and G. Steigman, "Constraining the early-Universe baryon density and expansion rate," Journal of Cosmology and Astroparticle Physics, vol. 2008, no. 6, article 16, 2008.

[25] R. V. Wagoner, W. A. Fowler, and F. Hoyle, "On the synthesis of elements at very high temperatures," The Astrophysical Journal, vol. 148, p. 3, 1967.
[26] M. S. Smith, L. H. Kawano, and R. A. Malaney, "Experimental, computational, and observational analysis of primordial nucleosynthesis," The Astrophysical Journal, Supplement Series, vol. 85, no. 2, pp. 219-247, 1993.

[27] R. Esmailzadeh, G. D. Starkman, and S. Dimopoulos, "Primordial nucleosynthesis without a computer," Astrophysical Journal Letters, vol. 378, no. 2, pp. 504-518, 1991.

[28] V. Mukhanov, The Physical Foundation of Cosmology, Cambridge University Press, Cambridge, Mass, USA, 2005.

[29] J. P. Kneller and G. Steigman, "BBN for pedestrians," New Journal of Physics, vol. 6, article 117, 2004.

[30] G. Steigman, "Primordial nucleosynthesis in the precision cosmology era," Annual Review of Nuclear and Particle Science, vol. 57, pp. 463-491, 2007.

[31] E. Komatsu, K. M. Smith, J. Dunkley (WMAP Collaboration) et al., "Seven-year Wilkinson microwave anisotropy probe (WMAP*) observations: cosmological interpretation," The Astrophysical Journal Supplement Series, vol. 192, no. 2, article 18, 2011.

[32] B. E. J. Pagel, E. A. Simonson, R. J. Terlevich, and M. G. Edmunds, "The primordial helium abundance from observations of extragalactic HII regions," Monthly Notices of the Royal Astronomical Society, vol. 255, pp. 325-345, 1992.

[33] Y. I. Izotov and T. X. Thuan, "The primordial abundance of 4He: evidence for non-standard big bang nucleosynthesis," Astrophysical Journal Letters, vol. 710, no. 1, pp. L67-L71, 2010.

[34] E. Aver, K. A. Olive, and E. D. Skillman, "A new approach to systematic uncertainties and self-consistency in helium abundance determinations," Journal of Cosmology and Astroparticle Physics, vol. 2010, no. 5, article 3, 2010.

[35] D. Kirkman, D. Tytler, N. Suzuki, J. M. O’Meara, and D. Lubin, "The cosmological baryon density from the deuteriumto-hydrogen ratio in QSO absorption systems: D/H toward Q1243+3047," The Astrophysical Journal, Supplement Series, vol. 149, no. 1, pp. 1-28, 2003.

[36] J. M. O’Meara, S. Burles, J. X. Prochaska, G. E. Prochter, R. A. Bernstein, and K. M. Burgess, "The deuterium-to-hydrogen abundance ratio toward the QSO SDSS J155810.16-003120.0 1," Astrophysical Journal Letters, vol. 649, no. 2, pp. L61-L65, 2006.

[37] M. Pettini, B. J. Zych, M. T. Murphy, A. Lewis, and C. C. Steidel, "Deuterium abundance in the most metal-poor damped Lyman alpha system: converging on $\Omega_{b, 0} h^{2}$," Monthly Notices of the Royal Astronomical Society, vol. 391, no. 4, pp. 1499-1510, 2008.

[38] M. Fumagalli, J. M. O’Meara, and J. X. Prochaska, “Detection of pristine gas two billion years after the big bang," Science, vol. 334, no. 6060, pp. 1245-1249, 2011.

[39] M. Pettini and R. Cooke, "A new, precise measurement of the primordial abundance of Deuterium," http://arxiv.org/pdf/1205.3785.pdf.

[40] F. Iocco, G. Mangano, G. Miele, O. Pisanti, and P. D. Serpico, "Primordial nucleosynthesis: from precision cosmology to fundamental physics," Physics Reports, vol. 472, no. 1-6, pp. 1-76, 2009.

[41] G. Steigman, "Primordial nucleosynthesis: successes and challenges," International Journal of Modern Physics E, vol. 15, no. 1, pp. 1-36, 2006.

[42] B. D. Fields, “The primordial lithium problem," Annual Review of Nuclear and Particle Science, vol. 61, pp. 47-68, 2011.

[43] M. Asplund, D. L. Lambertm, P. E. Nissen et al., "Lithium isotopic abundances in metal-poor halo stars," The Astrophysical Journal, vol. 644, p. 229, 2006. 
[44] S. G. Ryan, T. C. Beers, K. A. Olive, B. D. Fields, and J. E. Norris, "Primordial lithium and big bang nucleosynthesis," The Astrophysical Journal Letters, vol. 530, pp. L57-L60, 2000.

[45] T. Damour and B. Pichon, "Big bang nucleosynthesis and tensor-scalar gravity," Physical Review D, vol. 59, Article ID 123502, 1999.

[46] A. Coc, K. A. Olive, J. P. Uzan, and E. Vangioni, "Big bang nucleosynthesis constraints on scalar-tensor theories of gravity," Physical Review D, vol. 73, Article ID 083525, 2006.

[47] J. A. R. Cembranos, K. A. Olive, M. Peloso, and J.-P. Uzan, "Quantum corrections to the cosmological evolution of conformally coupled fields," Journal of Cosmology and Astroparticle Physics, vol. 907, article 025, 2009. 

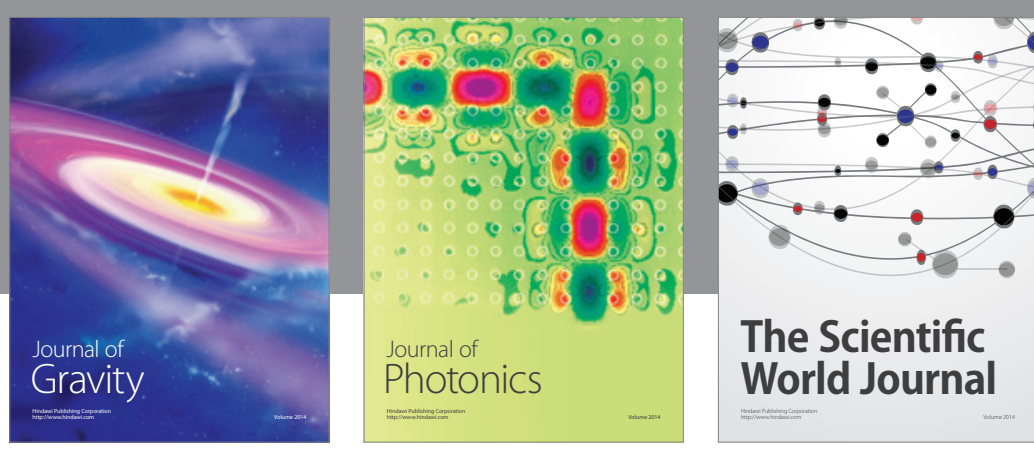

The Scientific World Journal
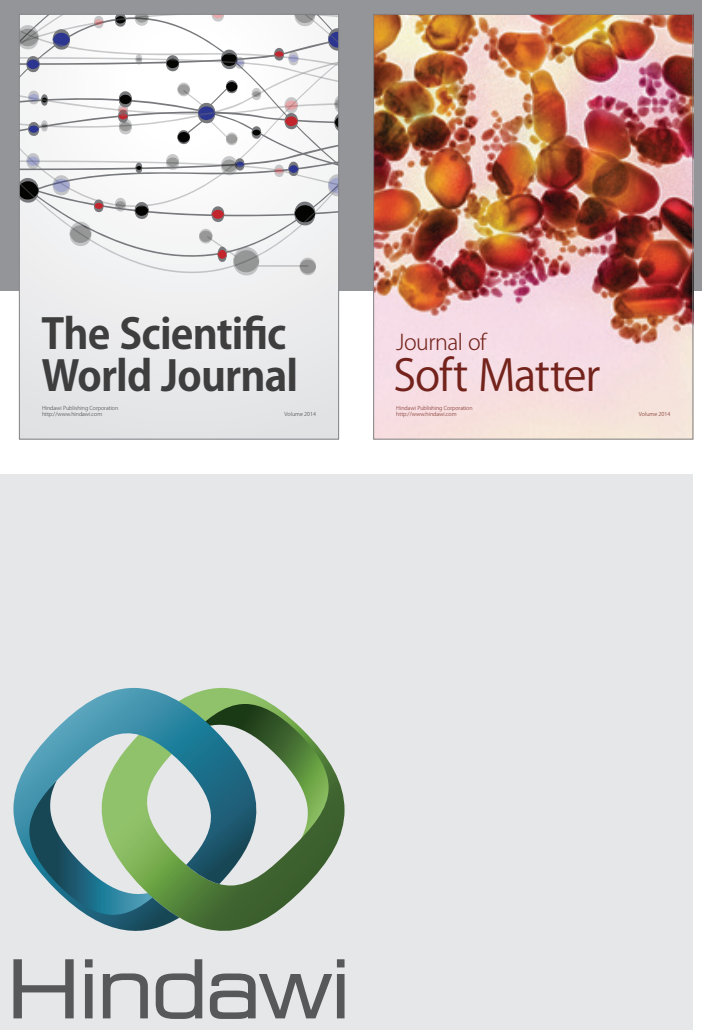

Submit your manuscripts at

http://www.hindawi.com

nternational Journal of

Statistical Mechanics
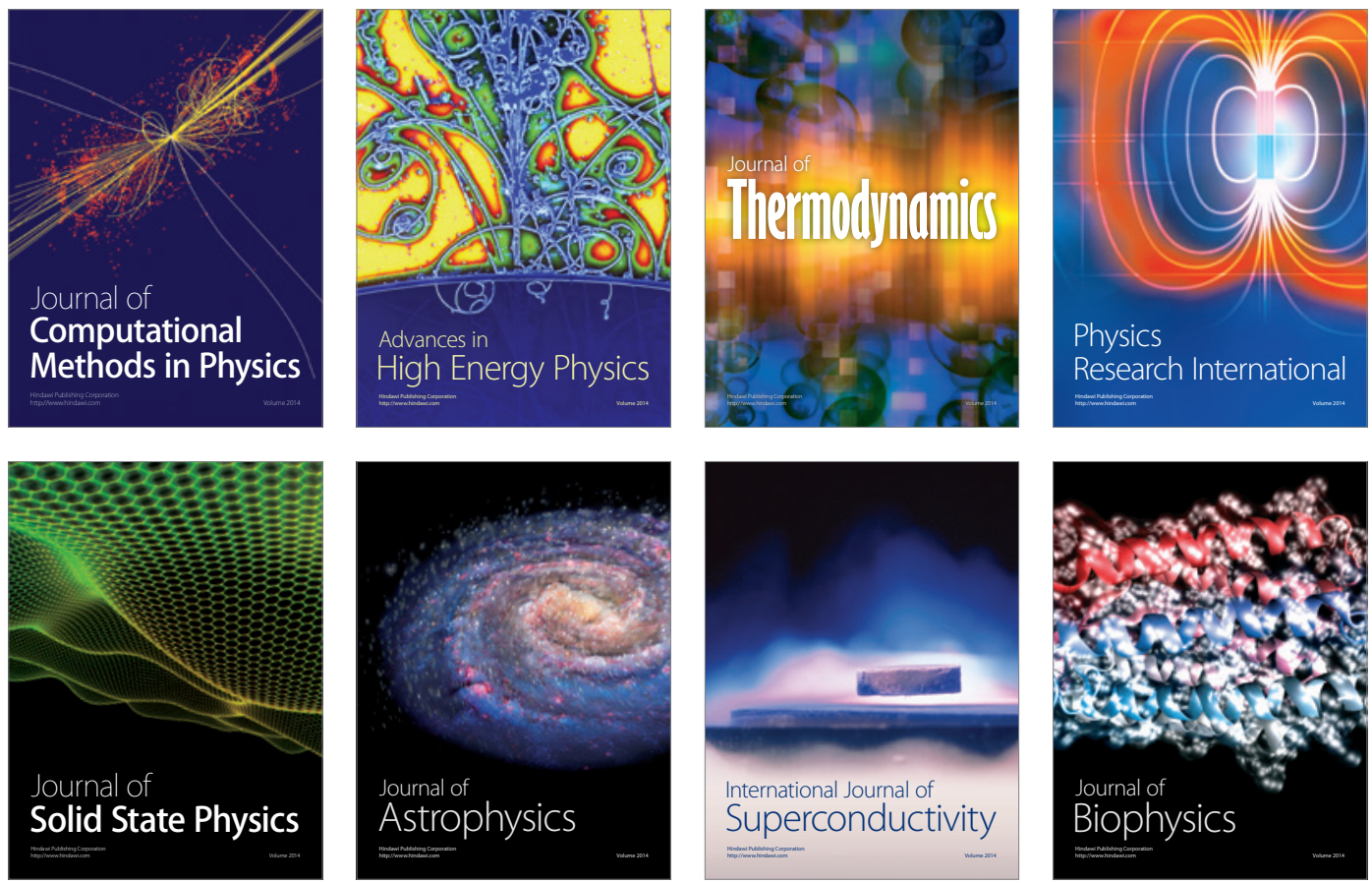
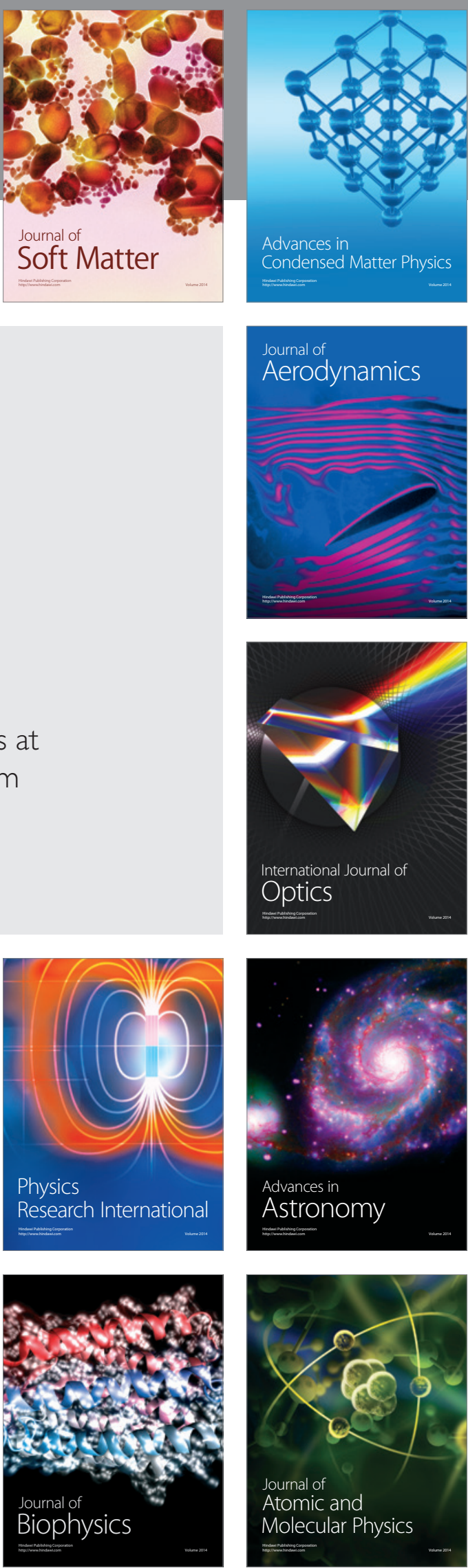